\title{
Primary Effusion Lymphoma in an Elderly HIV-Negative Patient with Hemodialysis: Importance of Evaluation for Pleural Effusion in Patients Receiving Hemodialysis
}

\author{
Yosuke Sasaki $^{\mathrm{a}}$ Takuya Isegawa $^{\mathrm{a}}$ Akira Shimabukuro $^{\mathrm{a}}$ \\ ${\text { Tomoki Yonahac }{ }^{c} \text { Hiroyasu Yonaha }}^{b}$ \\ Departments of ${ }^{\mathrm{a}}$ Internal Medicine and ${ }^{\mathrm{b}}$ General Medicine, Okinawa Yaeyama Hospital, \\ and 'Yonaha Medical Clinic, Okinawa, Japan
}

\section{Key Words}

Primary effusion lymphoma - Thoracentesis · Kaposi's sarcoma herpes virus/human herpes virus 8-negative effusion-based lymphoma - Primary effusion lymphoma-like lymphoma . Hemodialysis · Human herpes virus 8

\begin{abstract}
Pleural effusion is a ubiquitous complication in hemodialysis (HD) patients. Common etiologies of pleural effusion in this patient group are heart failure, volume overload, parapneumonic effusion, tuberculotic pleuritis, and uremic pleuritis. Although thoracentesis is a useful diagnostic method of pleural effusion, empirical reduction of the dry weight is often attempted without thoracentesis because pleural effusion is commonly caused by volume overload and responds to the dry-weight reduction. However, this empiricism has a risk of overlooking or delaying the diagnosis of potentially fatal etiologies that need specific treatments. We report an 86-year-old human immunodeficiency virus (HIV)-negative male on HD with primary effusion lymphoma (PEL), a large-cell non-Hodgkin lymphoma presenting with characteristic lymphomatous effusions in the absence of solid tumor masses, which is in association with human herpes virus 8 (HHV8) infection in immunocompromised individuals. The patient presented with left-sided pleural effusion. This is the first case report of PEL developing in a patient receiving HD. Thoracentesis and cytological analysis of the effusion was key to the diagnosis. We also review the literature regarding pleural effusion in HD patients. Further, we examine Kaposi's sarcoma herpes virus/HHV8-negative effusion-based
\end{abstract}


lymphoma, a newly proposed distinct lymphoma that clinically and cytomorphologically resembles PEL, because it can be cured without chemotherapy. This report may arouse clinicians' attention regarding the importance of evaluation for pleural effusion in HD patients, especially when the effusion or symptoms associated with pleural effusion are refractory to volume control.

(c) 2014 S. Karger AG, Basel

\section{Introduction}

Pleural effusion is a ubiquitous complication in hemodialysis (HD) patients [1]. Common etiologies of pleural effusion in this patient group are heart failure, volume overload, parapneumonic effusion, tuberculotic pleuritis, and uremic pleuritis [2]. Because of the frequency with which this complication is associated with volume overload or heart failure, empirical reduction of the dry weight (DW) is often attempted without thoracentesis [1]. Primary effusion lymphoma (PEL) is a large-cell non-Hodgkin lymphoma that presents with characteristic lymphomatous effusions in the absence of solid tumor masses and in association with human herpes virus 8 (HHV8) infection in immunocompromised individuals, especially patients with human immunodeficiency virus (HIV) infection, and rarely in the elderly [3]. Herein, we report an 86-year-old HIV-negative male on HD with PEL who presented with refractory left-sided pleural effusion as the first case report of PEL developing in a patient receiving HD.

\section{Case Report}

An 86-year-old man on HD visited the emergency department (ED) of Okinawa Yaeyama Hospital with the complaint of worsening dyspnea. The symptom had started with mild shortness of breath on exertion several months ago and had gradually exacerbated. Given the frequency of dyspnea associated with volume overload and/or heart failure in HD patients, his doctor in the HD clinic assumed that the symptom was caused by heart failure associated with volume overload and attempted to empirically reduce his DW. The doctor failed to reduce his DW because of the frequent falling of the blood pressure during HD. Furthermore, while his dyspnea became worse, the doctor had to increase his DW by as much as 2 kg over 3 weeks to prevent blood pressure falling and leg cramping during HD. The patient visited our ED with the complaint of an acute worsening of dyspnea during the previous few days. He denied having chest pain, cough, sputum, orthopnea or paroxysmal nocturnal dyspnea, fever, chills, night sweats, or anorexia. He had a past medical history of end-stage renal disease (ESRD) due to unknown etiology and was on scheduled HD ( $4 \mathrm{~h}$ per day at 3 days per week) for 4 years with an uneventful course. He also had well-controlled essential hypertension and chronic atrial fibrillation which was treated by anticoagulation with warfarin for 4 years. He developed a hemorrhagic gastric ulcer that was probably associated with excessive anticoagulation 1 year ago. Helicobacter pylori was serologically negative at that time. Surgical repair of the left inguinal hernia was performed 10 years ago. He had no previous history of tuberculosis, pneumonia, or heart failure. He was not diabetic. He lived with his family. He worked as a construction worker up until several years ago. He smoked 2 packs of cigarettes a day for 30 years up until 30 years ago. He had no habit of regular alcohol consumption. Family history was noncontributory.

On physical examination, he was alert and appeared slightly sick. His body weight at the ED was $46.1 \mathrm{~kg}$. His recent DW was $46.0 \mathrm{~kg}$ with an increase of $2 \mathrm{~kg}$ over 3 weeks. His blood 
pressure was $130 / 60 \mathrm{~mm} \mathrm{Hg}$, pulse was irregularly irregular with a rate of 50 beats per minute, respiration rate was 18 per minute, and body temperature was $37.4^{\circ} \mathrm{C}$. Conjunctivae were pale. Cervical examination revealed nondistended jugular veins and no lymphadenopathy. Heart sounds were irregularly irregular without murmur or friction rubs. Chest auscultation revealed remarkably diminished breath sounds at the left thorax. A dull sound was heard at the left thorax on percussion. The abdomen was benign. There was no cyanosis, clubbing, or edema at the extremities. An arteriovenous fistula at his left forearm was unremarkable. Chest X-ray (CXR) showed massive left-sided pleural effusion without findings of pulmonary congestion or masses. (A CXR performed 7 months previous at the HD clinic showed no pleural effusion.) Immediate transthoracic echocardiogram showed no evidence of acute heart failure or volume overload. Laboratory findings are listed in table 1. Blood examination was remarkable for monocytosis (17.4\%), hypoalbuminemia $(2.5 \mathrm{mg} / \mathrm{dl})$, elevated serum thyroid stimulating hormone over $10 \mu \mathrm{g} / \mathrm{ml}$ with positive anti-thyroid peroxidase antibody, and positive result for QuantiFERON-TB2G testing. Tumor marker testing revealed markedly elevated soluble interleukin-2 receptor. Thoracentesis with continuous pleural effusion drainage was immediately performed. The pleural effusion was grossly bloody and exudative with atypical cells, massive mesothelial pleocytosis, and elevation of lactate dehydrogenase (table 2). The cytology of the effusion was class IV with strong suspicion of malignant lymphoma. Both fluorescent stain and Ziehl-Neelsen stain of the effusion detected no acid-fast bacilli. The culture of the effusion was negative. Computed tomography scans with contrast material of the neck, thorax, abdomen, and pelvis detected no findings suggesting a primary lesion of the malignant tumor or lymphadenopathy. Given the massive malignant pleural effusion at the unilateral side in the absence of solid tumor masses, we suspected PEL of the left thoracic cavity. We submitted cell surface marker testing and immunostaining for HHV8 of the effusion. CD45, CD38, and CD138 were dominantly detected as cell surface markers. HHV8 was detected by specific immunostaining. Enzyme-linked immunoassay (ELISA) for HIV was negative. Based on these results, we diagnosed the patient with PEL.

The patient's dyspnea subsided after the drainage. More than 6 liters of effusion were extracted over the first 3 days. We removed the chest tube on the 5th hospital day. We also prescribed $25 \mu \mathrm{g}$ per day of levothyroxine for subclinical hypothyroidism due to Hashimoto thyroiditis, although the association with pleural effusion is unlikely. We notified his daughter of the diagnosis and prognosis and discussed his management. His family wanted no further invasive treatment or evaluation. With consideration of his age, underlying illness such as ESRD, the lack of established treatment for PEL, and the request from his family members, we gave up treating him with chemotherapy. As per the family's wishes, his daughter informed the patient of the diagnosis. We treated the patient with the goal of living at home with his family comfortably. He was discharged 2 weeks following his first admission. A CXR on the date of discharge revealed that there was no further accumulation of effusion. HD was continued at the HD clinic.

The patient was referred to our ED 1 month after the previous discharge with the complaint of dyspnea and left pleural effusion. After discontinuation of warfarin and administration of vitamin $\mathrm{K}$, we performed another thoracic tube placement. Although he consequently developed re-expansion pulmonary edema (which presented as dyspnea with bilateral wheeze, crackles, and symmetric pulmonary edema on CXR) that required noninvasive positive pressure ventilation (NPPV) and bacteremia with Enterobacter cloacae treated with intravenous ceftriaxone (CTRX) for 14 days, his symptoms eventually subsided. Given the symptomatic accumulation of effusion after less than 1 month, we performed pleurodesis with $5 \mathrm{~g}$ of talc to the left thorax. He was discharged again after 1 month hospitalization. 
Three months following the second admission, he visited the ED due to general fatigue. He was evaluated for anemia (hemoglobin $7.6 \mathrm{~g} / \mathrm{dl}$ ) and was transfused with the diagnosis of mixed anemia of chronic disease due to PEL and renal anemia. His condition was fully improved with transfusion and rehabilitation and he was discharged after 10 days hospitalization.

Two weeks following the previous hospitalization, he was referred to the ED due to acute hypoxia following a productive cough for 2 days. We treated him with intravenous CTRX 1 g every $12 \mathrm{~h}$, intravenous ciprofloxacin $200 \mathrm{mg}$ every $12 \mathrm{~h}$, intravenous nitroglycerin, NPPV, and emergent HD with the extracorporeal ultrafiltration method for clinical diagnosis of pneumonia combined with acute heart failure. Despite our intensive treatment, his condition rapidly deteriorated. Considering his underlying condition and the pain associated with treatment, we started intravenous morphine for palliation. He died peacefully the day after admission, 7 months after his diagnosis of PEL.

\section{Discussion}

PEL is a large-cell non-Hodgkin lymphoma presenting with characteristic lymphomatous effusions in the absence of solid tumor masses. PEL was first recognized as a distinct entity in the World Health Organization's classification of neoplastic diseases of the hematopoietic and lymphoid tissues in 2001. It is characteristically associated with HHV8 infection, which has been implicated in the pathogenesis of PEL. It encodes numerous genes involved in inducing cell proliferation and inhibiting apoptosis. Co-infection with EpsteinBarr virus (EBV) is commonly found, though EBV may not be pathogenic for PEL [3].

Histologically, PEL typically displays a 'null' lymphocyte phenotype: CD45 is expressed, but common primary pan-B-cell (CD19, CD20, CD79a, surface immunoglobulins) and T-cell (CD3, CD4, CD8) markers are absent. Instead, however, markers of lymphocyte activation (CD30, CD38, CD71, epithelial membrane antigen, human leukocyte antigen-DR) and plasma cell differentiation (CD138) are often present. Bcl-6 is usually absent. Definitive diagnosis hinges on the detection of viral infection by HHV8 in the neoplastic cells [3].

At first, PEL was considered to be associated with HIV infection because the majority of cases occur in young to middle-aged males, either homosexual or bisexual, with HIV infection. However, it can also occur in HIV-negative individuals who are immunocompromised as a result of solid-organ transplantation or cirrhosis. In rare instances, PEL can affect elderly individuals who are otherwise immunocompetent, especially those who live in geographic areas with high HHV8 prevalence, such as the Mediterranean region [3]. Even in Japan, where HHV8 prevalence is not high, several cases of PEL in elderly patients have been reported, especially in patients with malignant solid tumors [4, 5]. According to these reports, a combination of advanced age and immunocompromised status such as that arising from malignant solid tumors can be regarded as a risk factor of PEL. Given that it has also been considered that HD results in an immunocompromised status [6], a combination of advanced age and HD can predispose individuals to PEL. So far, this has been the first case report of PEL developing in an elderly patient receiving HD.

There is no standard treatment for PEL at present. Standard chemotherapy regimens for non-Hodgkin lymphoma (e.g., cyclophosphamide, doxorubicin, vincristine, and prednisolone) have been attempted with poor outcome (median survival is less than 6 months) [3]. Our patients survived for 7 months after diagnosis, which seems to be a common clinical outcome for cases of PEL. 
It is important to mention Kaposi's sarcoma herpes virus/HHV8-negative effusion-based lymphoma (KSHV/HHV8-negative EBL), also called HHV8-negative PEL or PEL-like lymphoma, a newly proposed distinct entity. KSHV/HHV8-negative EBL is a large B-cell lymphoma with lymphomatous effusions without association with HHV8 infection expressing pan-B-cell markers. This lymphoma is usually seen in fluid overload states and has been reported to have good prognosis when compared to PEL [7]. Although this lymphoma has been reported as a subtype of PEL without association with HHV8 infection because of its clinical and cytomorphological similarity with PEL, this entity is distinguished from PEL by three points: the lack of association with HHV8 infection, the association with fluid overload status, and the prognosis [7]. KSHV/HHV8-negative EBL is characteristically seen in individuals with an underlying medical condition such as cirrhosis or heart failure, and some cases have been effectively treated with pleural aspiration without chemotherapy $[7,8]$. This good response to pleural effusion eradication may explain the better outcome of KSHV/HHV8-negative EBL compared to PEL. Considering that fluid overload is common in HD patients, making the distinction between PEL and KSHV/HHV8-negative EBL by evaluating HHV8 infection is very important because the prognosis and etiologies differ. In this case, positive HHV8 immunostaining, resistance to pleurodesis and repeated pleural drainage, and short survival interval support a diagnosis of PEL.

Approximately $20 \%$ of hospitalized patients receiving HD develop pleural effusion [1]. In addition to common causes of pleural effusion in the general population such as parapneumonic effusion or heart failure, pleural effusion due to fluid overload, tuberculotic pleuritis, and uremic pleuritis are also common in patients receiving HD. Tuberculotic pleuritis has been an especially important issue for clinicians because of its public health implications. Uremic pleuritis is a unique etiology for HD patients. Although it is classically thought of as pleural effusion in patients with ESRD without HD, it has recently been reported that uremic pleuritis exists even in patients already receiving HD and is underestimated [2]. A recent study reported that uremic pleuritis comprised $40 \%$ of exudative effusion and was the most common etiology of exudative effusion [1]. Because uremic pleuritis responds to improvement in HD, this entity is now gathering clinical attention. Even pleural effusion due to heart failure is unique. Cases of isolated pleural effusion due to highoutput heart failure as a result of a giant vascular access have been reported $[9,10]$. Due to these common, unique, and important etiologies for this patient group, the prevalence and impact of malignant pleural effusion in HD patients may be less than that of the general population.

In spite of the fact that thoracentesis and analysis of the pleural effusion is a swift and useful method to distinguish the etiology of effusion in most cases, thoracentesis seems to be infrequently performed in clinical settings. In an article analyzing 257 hospitalized patients with long-term HD, thoracentesis was performed in only 26.9\% [1]. Even in a report on HD patients admitted for pleural effusion, thoracentesis was performed in $86.7 \%$ [2]. The reason for the infrequency of thoracentesis is likely due to the fact that most pleural effusion, especially bilateral, is associated with fluid overload, heart failure, or uremic pleuritis and responds to the fluid reduction or improvement of the HD prescription. Approximately $70 \%$ of bilateral and $17 \%$ of unilateral effusion was caused by fluid overload [1]. Thus, in many cases, analysis of the effusion is unnecessary. However, clinicians should be aware that this empiricism involves a risk of overlooking or delaying the diagnosis of potentially fatal etiologies that require specific treatments such as infection or KSHV/HHV8-negative EBL. Even in incurable malignant effusions, recognizing the diagnosis and prognosis will aid patient care. Thus, this case report of PEL developing in an elderly patient receiving HD, which is a combination of risk factors of PEL, may arouse clinicians' attention regarding the 
Sasaki et al.: Primary Effusion Lymphoma in an Elderly HIV-Negative Patient with Hemodialysis: Importance of Evaluation for Pleural Effusion in Patients Receiving Hemodialysis

importance of evaluation for pleural effusion in HD patients, especially when the effusion or symptoms associated with the pleural effusion are refractory to the reduction of the DW. Further studies on pleural effusion in HD patients, PEL, and KSHV/HHV8-negative EBL are warranted.

\section{Acknowledgments}

This article is dedicated to the memory of the late Mr. Yusho Sasaki and the fathers who survived the tumultuous post-World War II era.

\section{Disclosure Statement} interest.

On behalf of all authors, the corresponding author states that there are no conflicts of

\section{References}

1 Bakirci T, Sasak G, Ozturk S, Akcay S, Sezer S, Haberal M: Pleural effusion in long-term hemodialysis patients. Transplant Proc 2007;39:889-891.

-2 Rashid-Farokhi F, Pourdowlat G, Nikoonia M-R, Behzadnia N, Kahkouee S, Nassiri A-A, et al: Uremic pleuritis in chronic hemodialysis patients. Hemodial Int 2013;17:94-100.

-3 Patel S, Xiao P: Primary effusion lymphoma. Arch Pathol Lab Med 2013;137:1152-1154.

4 Yoneda G, Umemura S, Itoh H, Serizawa A, Matsui N, Miyajima Y, et al: A case of primary effusion lymphoma associated with human herpes virus 8 in an elderly HIV-seronegative male. J Jpn Soc Clin Cytol $2005 ; 44: 240-244$.

5 Yoneda G, Umemura S, Ito H, Matsui N, Miyajima Y, Yasuda M, et al: Primary effusion lymphoma diagnosed by cytology of pleural effusion and pericardial effusion. J Jpn Soc Clin Cytol 2004;43(suppl 2):467.

-6 De Marie S: Diseases and drug-related interventions affecting host defence. Eur J Clin Microbiol Infect Dis 1993;12(suppl 1):S36-S41.

7 Alexanian S, Said J, Lones M, Pullarkat ST: KSHV/HHV8-negative effusion-based lymphoma, a distinct entity associated with fluid overload states. Am J Surg Pathol 2013;37:241-249.

-8 Terasaki Y, Yamamoto H, Kiyokawa H, Okumura H, Saito K, Ichinohasama R, et al: Disappearance of malignant cells by effusion drainage alone in two patients with HHV-8-unrelated HIV-negative primary effusion lymphoma-like lymphoma. Int J Hematol 2011;94:279-284.

-9 Dhawan V, Ariyamuthu V, Malhotra K, Dalal P, Bichu P, Dorairajan S: Isolated pleural effusion as a presentation of high cardiac output heart failure in a hemodialysis patient. Hemodial Int 2012;16(suppl 1):S54-S57.

10 Chapman F, Blackmur JP, Fotheringham J, Watson S, Whitworth C, Dhaun N: An uncommon cause of pleural effusions in a dialysis patient. Lancet 2012;380:186. 
Sasaki et al.: Primary Effusion Lymphoma in an Elderly HIV-Negative Patient with Hemodialysis: Importance of Evaluation for Pleural Effusion in Patients Receiving Hemodialysis

Table 1. Results of blood examination

\begin{tabular}{ll}
\hline Leukocyte, /mm ${ }^{3}$ & 7,020 \\
$\quad$ Neutrophil, \% & 53.8 \\
$\quad$ Lymphocyte, \% & 19.9 \\
$\quad$ Monocyte, \% & 17.4 \\
$\quad$ Eosinophil, \% & 8.3 \\
$\quad$ Basophil, \% & 0.6 \\
Hgb, g/dl & 10.2 \\
Hct, \% & 30.3 \\
PT-INR & 1.6 \\
aPTT/standard, s & $45.1 / 30.0$ \\
Sodium, mEq/l & 139 \\
Potassium, mEq/l & 4.1 \\
Chloride, mEq/l & 105 \\
Calcium, mg/dl & 7.1 \\
Phosphorus, mg/dl & 5.1 \\
BUN, mg/dl & 40.9 \\
Creatinine, mg/dl & 4.8 \\
Glucose, mg/dl & 115 \\
AST, IU/l & 15 \\
ALT, IU/l & 9 \\
ALP, U/l & 275 \\
GGT, U/l & 16 \\
LDH, IU/l & 142 \\
CRP, mg/dl & 4.6 \\
Albumin, mg/dl & 2.5 \\
Total protein, mg/dl & 5 \\
BNP, pg/ml & 301 \\
TSH, $\mu$ IU/l & 15.4 \\
TPO Ab, U/ml & 6.6 \\
sIL-2R, U/ml & 6,981 \\
\hline
\end{tabular}

ALP = Alkaline phosphatase; ALT = alanine aminotransferase; $\mathrm{aPTT}=$ activated partial thromboplastin time; AST = aspartate aminotransferase; $\mathrm{BUN}=$ blood urea nitrogen; $\mathrm{CRP}=\mathrm{C}$-reactive protein; GGT $=\gamma$ glutamyl transpeptidase; $\mathrm{Hct}=$ hematocrit; $\mathrm{Hgb}=$ hemoglobin; $\mathrm{LDH}=$ lactate dehydrogenase; PT-INR = prothrombin time-international normalized ratio; sIL-2R = soluble interleukin-2 receptor; $\mathrm{TPO} A \mathrm{~b}=$ anti-thyroid peroxidase antibody; TSH = thyroid stimulating hormone. 
Sasaki et al.: Primary Effusion Lymphoma in an Elderly HIV-Negative Patient with Hemodialysis: Importance of Evaluation for Pleural Effusion in Patients Receiving Hemodialysis

Table 2. Results of pleural effusion analysis

$\begin{array}{ll}\text { Appearance } & \text { Bloody } \\ \text { Cell count, } / \mathrm{mm}^{3} & 10,360 \\ \quad \text { Mesothelial cell, \% } & 85 \\ \quad \text { Atypical cell, \% } & 10 \\ \quad \text { Lymphocyte, \% } & 4 \\ \quad \text { Eosinophil, \% } & 1 \\ \text { Red blood cell, } \times 1,000 / \mathrm{mm}^{3} & 610 \\ \text { Total protein, } \mathrm{mg} / \mathrm{dl} & 4.1 \\ \text { LDH, IU/l } & 5,257 \\ \text { Glucose, } \mathrm{mg} / \mathrm{dl} & 48 \\ \text { ADA, IU/l } & 113 \\ \text { Culture } & \text { Negative }\end{array}$

$\mathrm{ADA}=$ Adenosine deaminase; $\mathrm{LDH}=$ lactate dehydrogenase . 